\title{
High interleukin-8 and/or extracellular signal-regulated kinase 2 expression predicts poor prognosis in patients with hepatocellular carcinoma
}

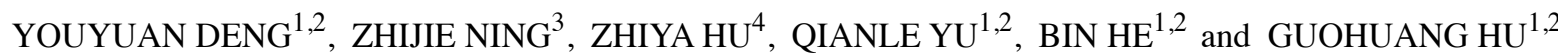

${ }^{1}$ Department of General Surgery and ${ }^{2}$ Institute of Digestive Surgery, Affiliated Changsha Hospital of Hunan Normal University, Changsha, Hunan $410006 ;{ }^{3}$ Department of Neurology, China-Japan Union Hospital of Jilin University, Changchun, Jilin 130013; ${ }^{4}$ Department of General Surgery, Third Hospital of Changsha, Changsha, Hunan 410015, P.R. China

Received April 10, 2019; Accepted August 29, 2019

DOI: 10.3892/ol.2019.10907

\begin{abstract}
Interleukin (IL)-8 and extracellular signal-regulated kinase (ERK) 2 play key roles in tumor progression, but the relationship between IL-8 and/or ERK2 expression in hepatocellular carcinoma (HCC) tissues and postoperative recurrence or survival is unclear. The expression levels of IL-8 and ERK2 in both HCC tissues and non-tumor liver tissues were analyzed using the Oncomine ${ }^{\mathrm{TM}}$ database and immunohistochemistry assay. Reverse transcription-quantitative PCR was then used to evaluate the expression levels of IL-8 and ERK2 in the tumor tissues of 67 patients with HCC undergoing radical hepatectomy. Pearson's correlation, Kaplan-Meier, Cox univariate and multivariate survival analyses were utilized to determine the correlation between IL-8 and ERK 2 expression in HCC tissues, and their potential prognostic significance. As indicated by the data from the Oncomine ${ }^{\mathrm{TM}}$ database, and the patient samples, IL-8 and ERK2 were expressed at significantly higher levels in HCC tissues than in non-tumor liver tissues $(\mathrm{P}<0.05)$. The rates of high IL-8 and ERK2 expression in HCC tissues were $43.28(29 / 67)$ and $34.33 \%$ (23/67), respectively, and the IL-8 and ERK2 expression levels were positively correlated $(\mathrm{r}=0.764 ; \mathrm{P}<0.001)$. Both ERK2 expression and IL-8/ERK2 co-expression were significantly associated with tumor size and differentiation $(\mathrm{P}<0.05)$. Additionally, high expression levels of IL-8, ERK2 and IL-8/ERK2 co-expression were all significantly associated with poor overall survival (OS; $\mathrm{P}<0.05$ ) and disease-free survival (DFS; $\mathrm{P}<0.05$ ). Multivariate Cox regression analysis also showed that high expression levels of IL-8, ERK2, and IL-8 and ERK2 were independent
\end{abstract}

Correspondence to: Professor Guohuang $\mathrm{Hu}$, Department of General Surgery, Affiliated Changsha Hospital of Hunan Normal University, 70 Lushan Road, Changsha, Hunan 410006, P.R. China E-mail: huguohuanghn@163.com

Key words: interleukin-8, extracellular signal-regulated kinase 2, immunohistochemistry, reverse transcription-quantitative PCR, hepatocellular carcinoma, prognosis prognostic factors for OS and DFS $(\mathrm{P}<0.05)$. The results of the present study indicate a significant increase in the risk of recurrence and mortality in HCC patients with high expression levels of IL-8 and/or ERK2, compared with patients with low expression. Therefore, IL-8 and ERK2 may be predictors of postoperative prognosis in patients with $\mathrm{HCC}$.

\section{Introduction}

Hepatocellular carcinoma (HCC) is one of the most prevalent primary malignancies of the liver (1). It is the fourth leading cause of cancer-associated mortality worldwide (2), and the third leading cause in China (3), where chronic hepatitis B virus infection and aflatoxin exposure are major risk factors (2). Surgical resection is the primary method of treating $\mathrm{HCC}$, but due to rapid disease progression, most patients exhibit extensive metastasis before the time of surgery. Moreover, only $20 \%$ of HCC patients undergo radical surgical resection (4); following resection, the 2 -year HCC recurrence rate is as high as 55\%, and most patients develop unresectable metastatic disease (5). The 5-year survival rate of HCC patients is $<50 \%$ (6). Therefore, identification of early indicator molecules for postoperative recurrence and survival, and the improvement of long-term survival, are urgently required.

Interleukins (ILs)-6, IL-8, IL-17 and IL-10, as well as other inflammation- or immunity-related cytokines, have received attention for their association with tumors. These cytokines can either promote or inhibit the development of tumors (7), and are critical for assessing the risk of postoperative recurrence and long-term survival. IL-8, also known as CXC chemokine 8, has received increasing interest as a tumor inflammatory factor. IL-8 is a member of the chemokine family (8) and plays an important role in the tumor microenvironment, influencing tumor progression and regulating neovascularization, tumor cell growth, apoptosis and cell migration $(9,10)$. It is expressed in a variety of tumors such as lung (11), breast (12) and colon cancer (13); a study by Wang et al (14) demonstrated that IL-8 expression is elevated in HCC. IL-8 promotes tumor cellular proliferation and neovascularization, either directly or indirectly, through tumor vascular endothelial cell receptors, thereby promoting tumor growth and metastasis $(14,15)$. 
IL-8 also induces the activation of the classical mitogen-activated protein kinase (MAPK) signaling cascade, and subsequent downstream phosphorylation of both extracellular signal-regulated kinase (ERK)1 and ERK2 in neutrophils and tumor cells (16). ERK1/2 are key members of the MAPK family, whose activation is closely associated with the occurrence and development of various tumors $(17,18)$. Furthermore, Schmitz et al (19) detected high expression levels of ERK1/2 in HCC tissues, and ERK1/2 activation in HCC also constitutes an independent prognostic factor. Although these data reveal a possible role for IL- 8 and ERK $1 / 2$ in tumor progression, the relationship between IL-8 and/or ERK2 expression in HCC tissues, and postoperative recurrence and survival, remains unclear.

To further study the relationship between IL-8 and/or ERK2 levels in HCC, and recurrence and survival after hepatectomy, the expression levels of IL-8 and ERK2 in non-tumor liver tissues and HCC tissues were determined using the Oncomine $^{\mathrm{TM}}$ database and immunohistochemistry (IHC). Subsequently, reverse transcription-quantitative (RT-q) PCR was used to quantify IL-8 and ERK2 expression in the tumor tissues of 67 patients with $\mathrm{HCC}$, and their relationship with HCC clinical pathological features was then determined. This in-depth study of the risk factors of HCC recurrence and survival provides a theoretical basis for improving the long-term survival of patients with HCC.

\section{Materials and methods}

Data retrieval from the Oncomine ${ }^{T M}$ database. To investigate the clinical importance of IL-8 and ERK2 in HCC, Oncomine ${ }^{\mathrm{TM}}$ [https://www.oncomine.org/resource/main.html; GSE14323 (GPL571) and GSE14520 (GPL3921)] was searched for published data to analyze the mRNA expression levels of IL-8 and ERK2 in HCC tissues and non-tumor liver tissues $(20,21)$.

Patient information. The use of patient samples in the present study was approved by The Hunan Normal University Medical Ethics Committee, and accords with the provisions stated in The Declaration of Helsinki, as revised in 2013. A total of 67 frozen HCC specimens were collected from patients who underwent surgery at the Department of General Surgery, Affiliated Changsha Hospital, Hunan Normal University between January 2002 and December 2012. An additional 60 paraffin-embedded HCC tissues and adjacent non-tumor liver tissues were collected. The inclusion criteria were: i) R0 tumor resection and ii) postoperative pathology confirmed as HCC. The exclusion criteria were: i) Administration of any anti-cancer treatment before surgery; ii) serious complications or death within 30 days post-surgery; iii) non-tumor related mortality; and iv) incomplete clinical, pathological or surgical data. The 67 HCC patients were aged between 36 and 83 years, with a median age of 55.0 years. There was a total of 55 men and 12 women, with a male-to-female ratio of 4.58:1. According to the TNM staging detailed in the eighth edition of the American Joint Committee on Cancer (AJCC) Cancer Staging Manual (22), 42 patients had stage I tumors, 18 had stage II tumors, and 7 had stage III tumors.

IHC. All patient specimens were fixed in $10 \%$ neutral formalin, embedded in paraffin, cut into $4-\mu \mathrm{m}$-thick serial sections and
Table I. Association between mRNA expression levels of IL-8 and ERK2.

\begin{tabular}{|c|c|c|c|c|}
\hline \multirow{2}{*}{$\begin{array}{l}\text { ERK2 } \\
\text { expression }\end{array}$} & \multicolumn{2}{|c|}{$\begin{array}{c}\text { IL-8 } \\
\text { expression }\end{array}$} & \multirow{2}{*}{$\begin{array}{c}\text { Pearson's contingency } \\
\text { coefficient }\end{array}$} & \multirow[b]{2}{*}{ P-value } \\
\hline & High & Low & & \\
\hline High & 22 & 1 & 0.764 & $<0.001^{\circ}$ \\
\hline Low & 7 & 37 & & \\
\hline
\end{tabular}

IL-8, interleukin-8; ERK2, extracellular regulated protein kinases 2. ${ }^{\mathrm{a}} \mathrm{P}<0.05$.

stained as previously described (23). The primary antibody against IL-8 was purchased from R\&D Systems, Inc., (1:500; cat. no. AF-208-NA), and the primary antibody against ERK2 was purchased from Santa Cruz Biotechnology, Inc., (1:200, cat. no. SC-1647). Expression levels were scored as the proportion of the immuno-positive staining area $(0,0 \% ; 1,1-25 \% ; 2$, 26-50\%; and 3, 51-100\%) multiplied by the staining intensity ( 0 , negative; 1 , low; 2 , medium; 3 , high), and ranged from 0 to 9 . The scores were independently evaluated by two pathologists.

$R T-q P C R$. Total RNA was extracted from the frozen HCC tissues using TRIzol $^{\circledR}$ (Invitrogen; Thermo Fisher Scientific, Inc.) according to the manufacturer's protocol. Subsequently, reverse transcription was performed using the PrimeScript ${ }^{\mathrm{TM}}$ RT kit (Takara Bio, Inc.) according to the manufacturer's protocols. SYBR Premix EX Taq ${ }^{\mathrm{TM}}$ (Takara Bio, Inc.) was used for qPCR (according to the manufacturer's protocol) on an ABI 7900 Prism HT (Applied Biosystems; Thermo Fisher Scientific, Inc.). Relative gene expression was quantified using the $2^{-\Delta \Delta \mathrm{Cq}}$ method (24), and the patients were divided into high- and low-IL-8 and -ERK2 expression groups using the median expression value as the cut-off point. The PCR primers were as follows: IL-8 forward, 5'-AAGAAACCACCGGAA GGAAC-3', and reverse, 5'-ACTCCTTGGCAAAACTGC AC-3'; ERK2 forward, 5'-GAAGGTGCCTACGGGATGG-3', and reverse, 5'-GGTCAATGGTTGGTGTGCGG-3'; and GAPDH forward, 5'-AACAGCCTCAAGATCATCAGCA-3', and reverse, 5'-CATGAGTCCTTCCACGATACCA-3'. The thermocycling conditions were as follows: Initial denaturation for $30 \mathrm{sec}$ at $98^{\circ} \mathrm{C}$, followed by 24 cycles of $98^{\circ} \mathrm{C}$ for $15 \mathrm{sec}$ and $72^{\circ} \mathrm{C}$ for $30 \mathrm{sec}$, and lastly $72^{\circ} \mathrm{C}$ for $5 \mathrm{~min}$ to allow final extension before cooling to $4^{\circ} \mathrm{C}$.

Case follow-up. Follow-ups in the form of outpatient visits or telephone calls were conducted for all cases that met the study criteria. The regular follow-up plan was as follows: i) Review every 3 months within 2 years after surgery; ii) review every 6 months after 2-5 years; and iii) review every year after 5 years. The review included an analysis of liver function, an abdominal ultrasound and a chest radiograph. If required, enhanced computed tomography, magnetic resonance imaging and needle biopsy were performed. Recurrence was defined as confirmation of the presence of new lesions in or outside of the liver via imaging studies or biopsy. Overall survival (OS) was defined as the time between the date of surgery to death 
A
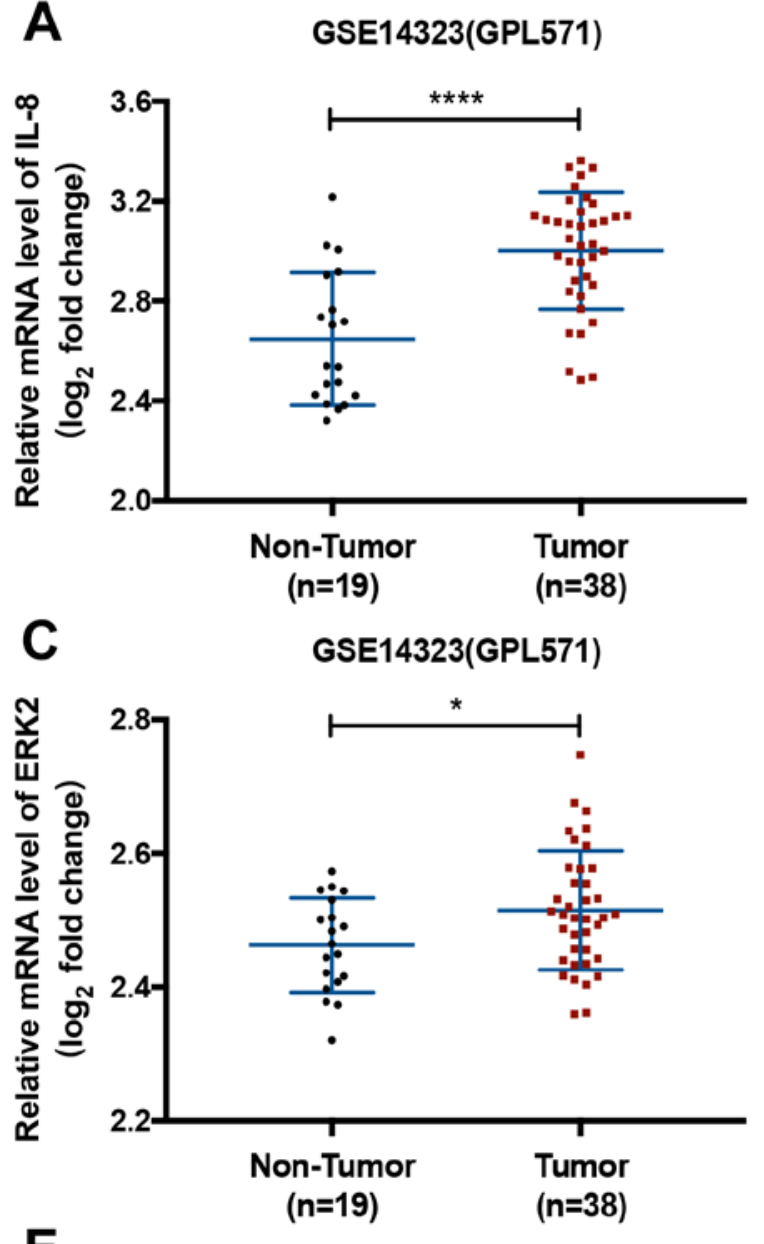

E

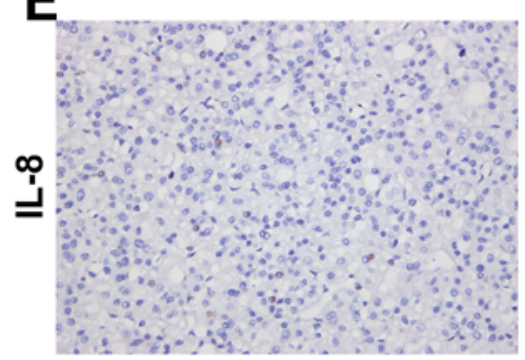

Non-Tumor (400X)

G

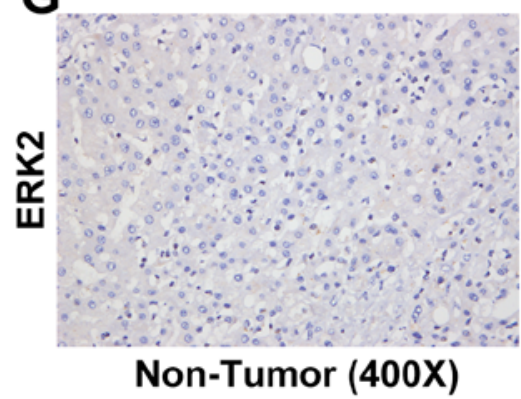

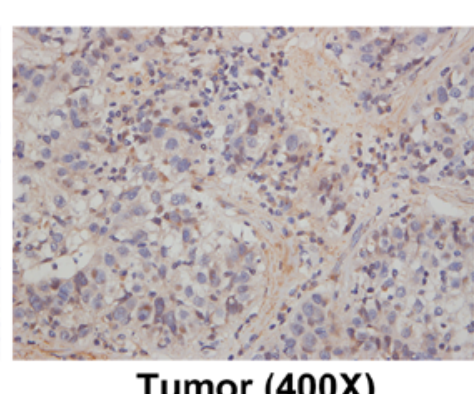

Tumor (400X)

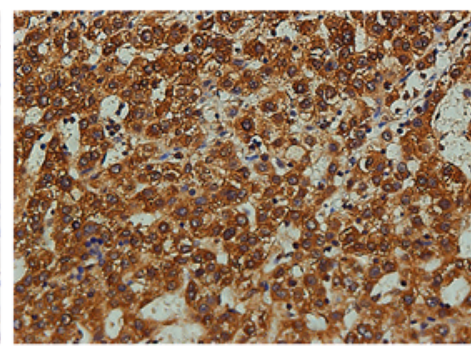

Tumor (400X)
B

GSE14520(GPL3921)

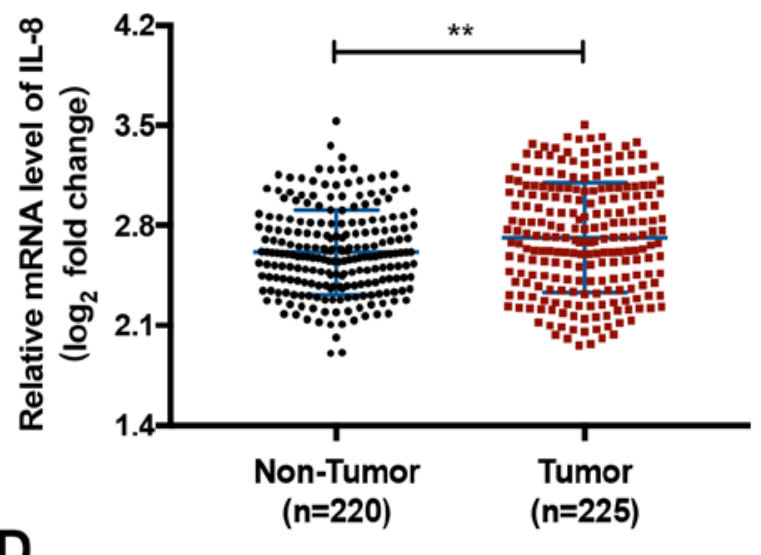

D

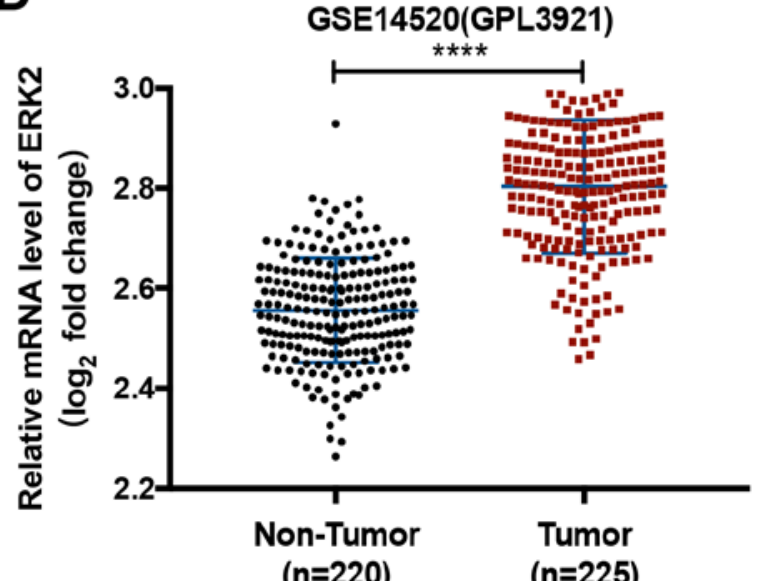

F

$(n=220)$
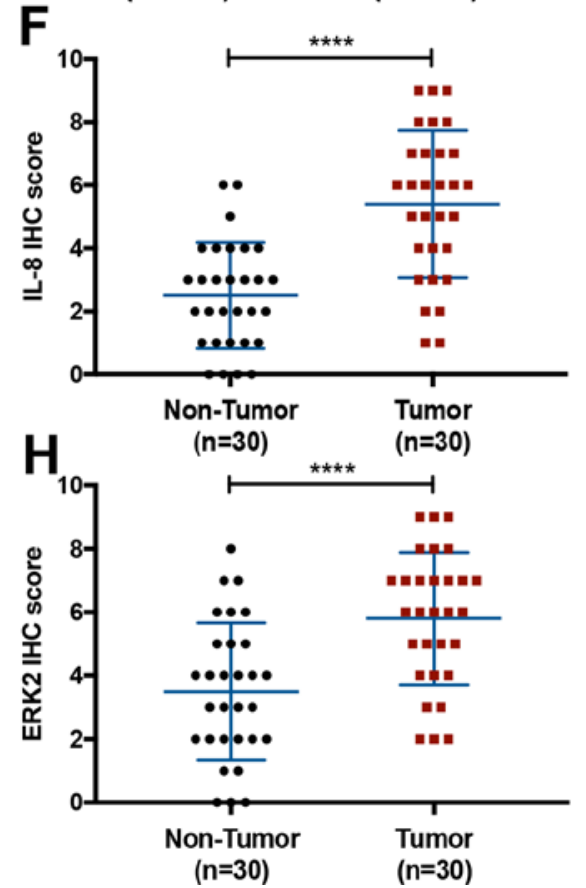

Figure 1. IL-8 and ERK2 expression in HCC patients. IL-8 mRNA expression level data in HCC vs. non-tumor liver tissues were extracted from the (A) GSE14323 (GPL571) and (B) GSE14520 (GPL3921) HCC datasets. ERK2 mRNA expression levels in HCC vs. non-tumor liver tissues were retrieved from the (C) GSE14323 (GPL571) and (D) GSE14520 (GPL3921) HCC datasets. Representative images of (E) IL-8 and (G) ERK2 protein expression. Quantitative analysis of (F) IL-8 and (H) ERK2 IHC score. IL-8, interleukin-8; ERK2, extracellular signal-regulated kinase 2; HCC, hepatocellular carcinoma; OS overall survival; DFS, disease-free survival; IHC, immunohistochemistry. ${ }^{*} \mathrm{P}<0.05,{ }^{* *} \mathrm{P}<0.01$ and ${ }^{* * * * *} \mathrm{P}<0.0001$

or follow-up, and disease-free survival (DFS) was defined as the date of surgery to relapse or follow-up. Both OS and DFS were calculated on a monthly basis, and the follow-up deadline was December 2018. 
Table II. Correlations of IL-8 and ERK2 mRNA expression with clinicopathological characteristics.

\begin{tabular}{|c|c|c|c|c|c|c|c|c|c|}
\hline \multirow[b]{2}{*}{ Parameter } & \multicolumn{2}{|c|}{ IL-8 } & \multirow[b]{2}{*}{ P-value } & \multicolumn{2}{|c|}{ ERK2 } & \multirow[b]{2}{*}{ P-value } & \multicolumn{2}{|c|}{ IL-8 and ERK2 } & \multirow[b]{2}{*}{ P-value } \\
\hline & High & Low & & High & Low & & Both high & Others & \\
\hline Sex & & & 0.158 & & & 0.678 & & & 0.765 \\
\hline Male & 26 & 29 & & 20 & 35 & & 19 & 36 & \\
\hline Female & 3 & 9 & & 3 & 9 & & 3 & 9 & \\
\hline Age & & & 0.372 & & & 0.940 & & & 0.747 \\
\hline$<50$, years & 7 & 13 & & 7 & 13 & & 6 & 14 & \\
\hline$\geq 50$, years & 22 & 25 & & 16 & 31 & & 16 & 31 & \\
\hline Alcoholism & & & 0.438 & & & 0.180 & & & 0.325 \\
\hline Absence & 18 & 27 & & 13 & 32 & & 13 & 32 & \\
\hline Presence & 11 & 11 & & 10 & 12 & & 9 & 13 & \\
\hline HBV & & & 0.623 & & & 1.000 & & & 1.000 \\
\hline Positive & 23 & 33 & & 19 & 37 & & 18 & 38 & \\
\hline Negative & 6 & 5 & & 4 & 7 & & 4 & 7 & \\
\hline Cirrhosis & & & 0.502 & & & 1.000 & & & 1.000 \\
\hline Absence & 0 & 2 & & 1 & 1 & & 0 & 2 & \\
\hline Presence & 29 & 36 & & 22 & 43 & & 22 & 43 & \\
\hline AFP & & & 0.660 & & & 0.634 & & & 0.958 \\
\hline$\leq 400 \mu \mathrm{g} / 1$ & 22 & 27 & & 16 & 33 & & 16 & 33 & \\
\hline$>400 \mu \mathrm{g} / 1$ & 7 & 11 & & 7 & 11 & & 6 & 12 & \\
\hline Albumin & & & 0.669 & & & 1.000 & & & 1.000 \\
\hline$<35 \mathrm{~g} / 1$ & 2 & 5 & & 2 & 5 & & 2 & 5 & \\
\hline$\geq 35 \mathrm{~g} / \mathrm{l}$ & 27 & 33 & & 21 & 39 & & 20 & 40 & \\
\hline ALT & & & 0.401 & & & 0.848 & & & 0.435 \\
\hline$\leq 60 \mathrm{U} / 1$ & 26 & 30 & & 20 & 36 & & 20 & 36 & \\
\hline$>60 \mathrm{U} / 1$ & 3 & 8 & & 3 & 8 & & 2 & 9 & \\
\hline AST & & & 0.839 & & & 0.923 & & & 0.728 \\
\hline$\leq 42 \mathrm{U} / 1$ & 22 & 28 & & 17 & 33 & & 17 & 33 & \\
\hline$>42 \mathrm{U} / 1$ & 7 & 10 & & 6 & 11 & & 5 & 12 & \\
\hline PLT & & & 0.352 & & & 0.923 & & & 0.803 \\
\hline$<100 \times 10^{9} / 1$ & 9 & 8 & & 6 & 11 & & 6 & 11 & \\
\hline$\geq 100 \times 10^{9} / 1$ & 20 & 30 & & 17 & 33 & & 16 & 34 & \\
\hline Bilirubin & & & 0.623 & & & 1.000 & & & 1.000 \\
\hline$\leq 22 \mu \mathrm{mol} / 1$ & 23 & 33 & & 19 & 37 & & 18 & 38 & \\
\hline$>22 \mu \mathrm{mol} / 1$ & 6 & 5 & & 4 & 7 & & 4 & 7 & \\
\hline Tumor number & & & 0.662 & & & 1.000 & & & 1.000 \\
\hline Single & 24 & 34 & & 20 & 38 & & 19 & 39 & \\
\hline Multiple & 5 & 4 & & 3 & 6 & & 3 & 6 & \\
\hline Tumor size & & & 0.117 & & & $0.013^{\mathrm{a}}$ & & & $0.019^{\mathrm{a}}$ \\
\hline$\leq 5 \mathrm{~cm}$ & 19 & 32 & & 13 & 38 & & 12 & 39 & \\
\hline$>5 \mathrm{~cm}$ & 9 & 6 & & 9 & 6 & & 9 & 6 & \\
\hline Tumor margin & & & 0.981 & & & 0.627 & & & 0.762 \\
\hline$\leq 2 \mathrm{~cm}$ & 10 & 13 & & 7 & 16 & & 7 & 16 & \\
\hline$>2 \mathrm{~cm}$ & 19 & 25 & & 16 & 28 & & 15 & 29 & \\
\hline Pathological differentiation & & & 0.134 & & & $0.014^{\mathrm{a}}$ & & & $0.008^{\mathrm{a}}$ \\
\hline High & 19 & 31 & & 13 & 37 & & 12 & 38 & \\
\hline Middle and low & 10 & 7 & & 10 & 7 & & 10 & 7 & \\
\hline $\begin{array}{l}\text { Microvascular tumor } \\
\text { thrombus }\end{array}$ & & & 0.469 & & & 0.524 & & & 0.415 \\
\hline Yes & 10 & 10 & & 8 & 12 & & 8 & 12 & \\
\hline No & 19 & 28 & & 15 & 32 & & 14 & 33 & \\
\hline
\end{tabular}


Table II. Continued.

\begin{tabular}{|c|c|c|c|c|c|c|c|c|c|}
\hline \multirow[b]{2}{*}{ Parameter } & \multicolumn{2}{|c|}{ IL-8 } & \multirow[b]{2}{*}{ P-value } & \multicolumn{2}{|c|}{ ERK2 } & \multirow[b]{2}{*}{ P-value } & \multicolumn{2}{|c|}{ IL-8 and ERK2 } & \multirow[b]{2}{*}{ P-value } \\
\hline & High & Low & & High & Low & & Both high & Others & \\
\hline Capsule invasion & & & 0.971 & & & 0.846 & & & 0.951 \\
\hline Yes & 23 & 30 & & 19 & 34 & & 18 & 35 & \\
\hline No & 6 & 8 & & 4 & 10 & & 4 & 10 & \\
\hline TNM stage & & & 0.236 & & & 0.078 & & & 0.061 \\
\hline $\mathrm{I}+\mathrm{II}$ & 24 & 36 & & 18 & 42 & & 17 & 43 & \\
\hline III & 5 & 2 & & 5 & 2 & & 5 & 2 & \\
\hline
\end{tabular}

IL-8, interleukin-8; ERK2, extracellular signal-regulated kinase 2; HBV, hepatitis B virus; AFP, alpha-fetoprotein; ALT, alanine aminotransferase; AST, alanine aminotransferase; PLT, platelet; TNM, tumor, lymph node, metastasis. ${ }^{\mathrm{a}} \mathrm{P}<0.05$.
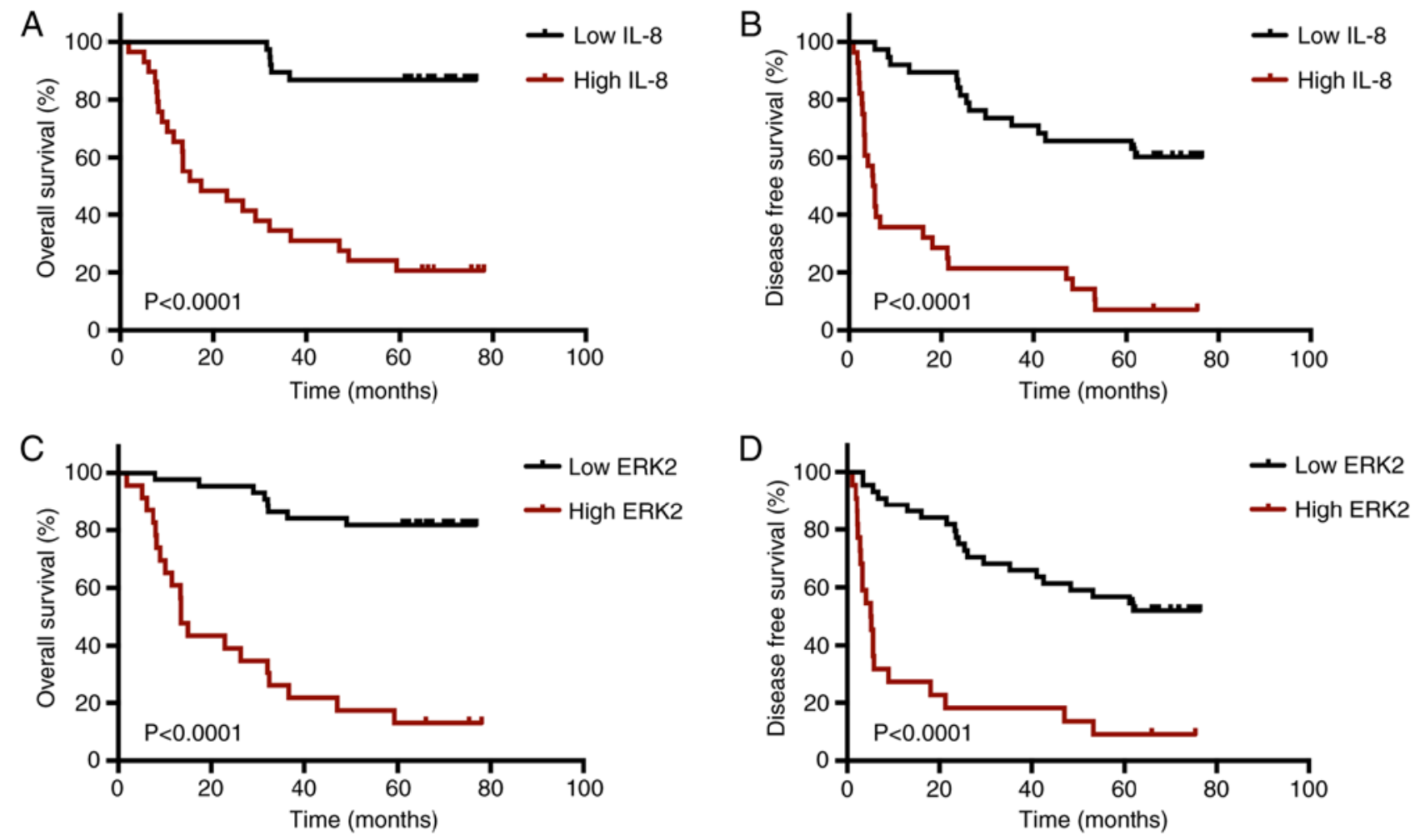

Figure 2. Prognostic values of IL-8 and ERK2 expression in HCC tissues. (A-D) Kaplan-Meier analysis of overall survival and disease-free survival among different tumor types stratified by (A and B) IL-8 and (C and D) ERK2. IL-8, interleukin-8; ERK2, extracellular signal-regulated kinase 2; HCC, hepatocellular carcinoma.

Statistical analysis. Statistical processing was performed using SPSS software v.19.0 (IBM Corp.). Spearman's correlation analysis was used to calculate the correlation between IL-8 and ERK2 expression levels. The $\chi^{2}$ test and Fisher's exact probability test were used to analyze the correlation between IL-8 and ERK2 expression in HCC tissues, and patient clinicopathological features. Survival analysis was performed using Kaplan-Meier curves, and the relationship between IL-8 and/or ERK2 expression and postoperative recurrence, and survival in patients with HCC was determined using the log-rank test. Univariate and multivariate analyses of $\mathrm{HCC}$ recurrence and survival were performed using the Cox proportional hazard model to screen for variables. The data are presented as the mean \pm standard deviation, and $\mathrm{P}<0.05$ was considered to indicate a statistically significant difference.

\section{Results}

Expression of IL-8 and ERK2 in non-tumor liver tissues and HCC tissues. The mRNA expression levels of IL-8 and ERK2 were retrieved from two published HCC datasets published in the Oncomine ${ }^{\mathrm{TM}}$ database. It was observed that the mRNA levels of both IL-8 and ERK2 were significantly higher in HCC tissues compared with non-tumor liver tissues from both datasets $(\mathrm{P}<0.05$; Fig. 1A-D). Subsequently, this result 
A

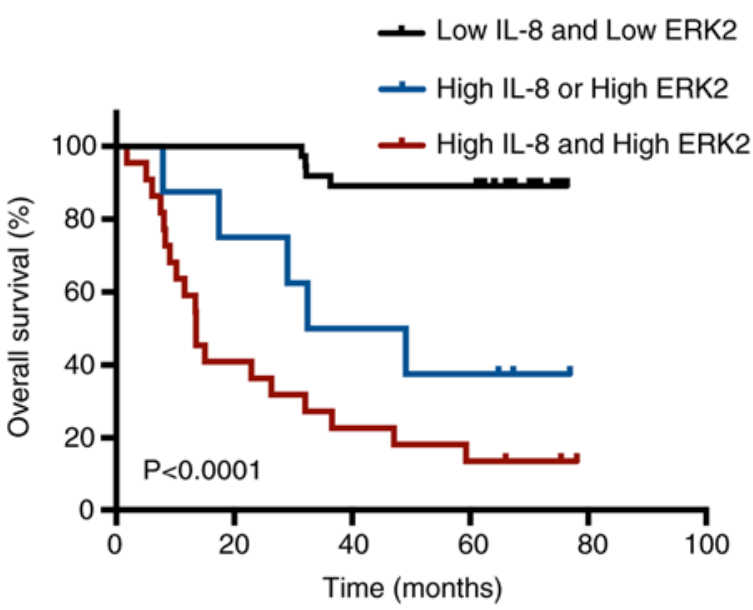

C

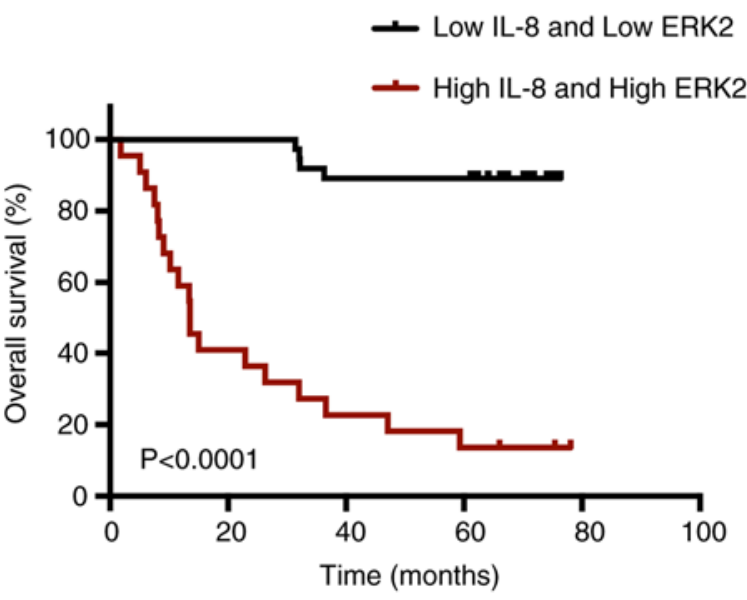

E

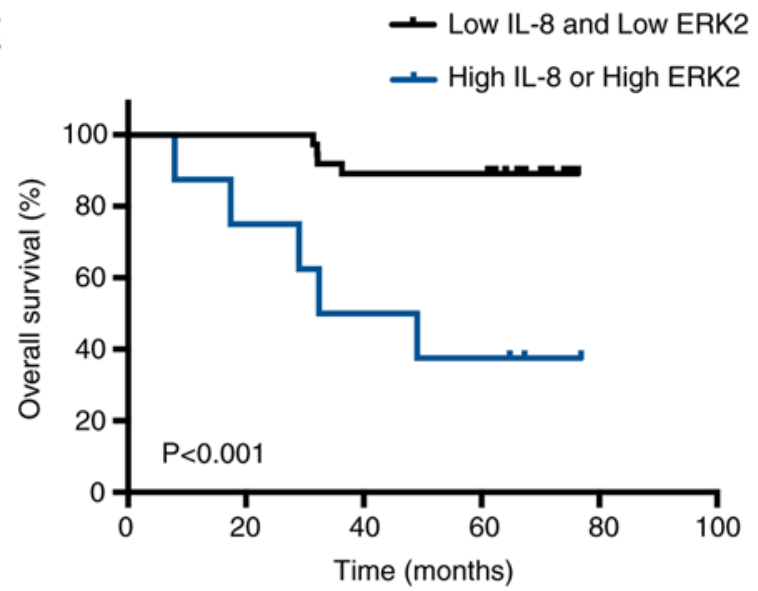

B

- Low IL-8 and Low ERK2
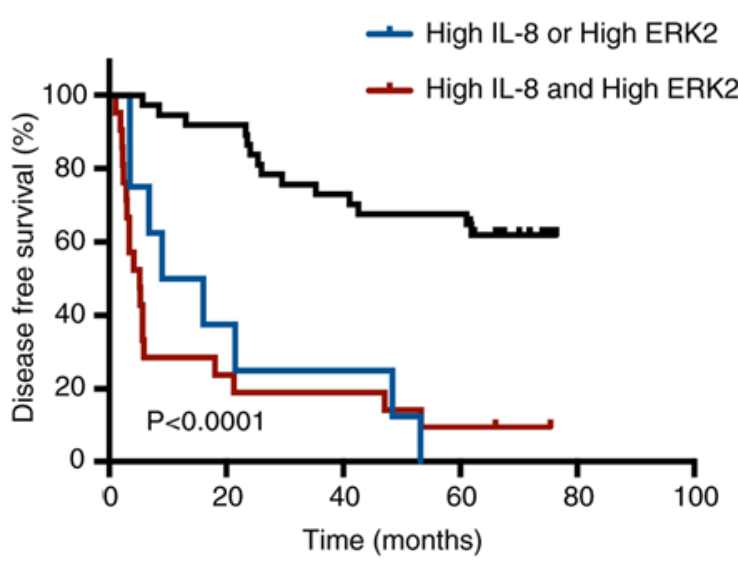

D

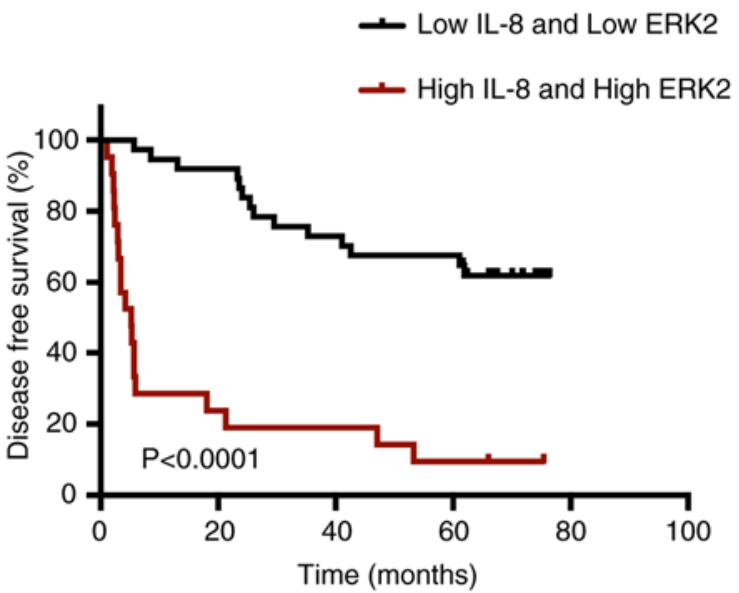

$\mathrm{F}$
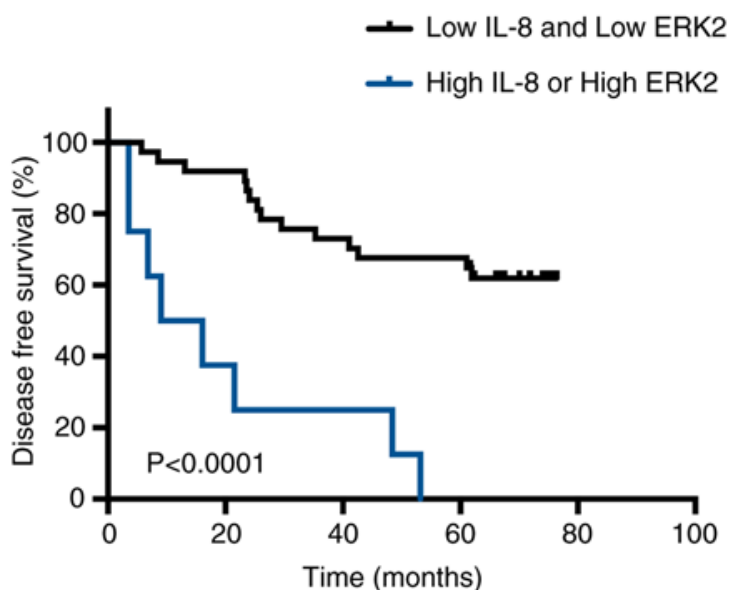

Figure 3. Prognostic values of IL-8 and/or ERK2 expression in HCC tissues. (A-F) Kaplan-Meier analysis of overall survival and disease-free survival among different tumor types stratified by IL-8 and/or ERK2 expression: Both high vs. all others (A and B) both high vs. both low (C and D) IL-8 or ERK2 high vs. both low (E and F) IL-8, interleukin-8; ERK2, extracellular signal-regulated kinase 2; HCC, hepatocellular carcinoma; OS overall survival; DFS, disease-free survival.

was validated in patient samples using IHC. The protein expression levels of IL-8 and ERK2 were significantly higher in HCC tissues compared with non-tumor liver tissues $(\mathrm{P}<0.05$; Fig. 1E-H).

Relationship between IL-8 and/or ERK2 expression and the clinicopathological features of HCC. IL-8 and ERK2 exhibited high expression rates in 43.28 (29/67) and $34.33 \%$ (23/67) of the HCC tissues, respectively. Additionally,
Pearson's correlation analysis indicated a positive correlation between IL-8 and ERK2 expression ( $\mathrm{r}=0.764 ; \mathrm{P}<0.001$; Table I). Although no significant correlation was observed between IL-8 expression and clinicopathological features in HCC tissues, ERK2 expression was significantly associated with both tumor size and differentiation $(\mathrm{P}<0.05$; Table II). Moreover, IL-8 and ERK 2 co-expression was also significantly associated with tumor size and differentiation $(\mathrm{P}<0.05$; Table II). 
Table III. Univariate analysis of variables with patient overall survival (Cox regression model).

\begin{tabular}{|c|c|c|c|}
\hline \multirow[b]{2}{*}{ Variable } & \multicolumn{3}{|c|}{ Univariate analysis } \\
\hline & Hazard ratio & $95 \% \mathrm{CI}$ & $\mathrm{P}$-value \\
\hline Sex (male vs. female) & 2.143 & $0.647-7.101$ & 0.212 \\
\hline Age $(\geq 50$ vs. $<50$, years $)$ & 1.046 & $0.460-2.375$ & 0.915 \\
\hline HBV (positive vs. negative) & 2.110 & $0.895-4.975$ & 0.088 \\
\hline Cirrhosis (present vs. absent) & 1.053 & $0.143-7.756$ & 0.960 \\
\hline $\operatorname{AFP}(>400 \mu \mathrm{g} / 1 \mathrm{vs} . \leq 400 \mu \mathrm{g} / \mathrm{l})$ & 1.205 & $0.531-2.737$ & 0.655 \\
\hline Albumin (>35 g/l vs. $\geq 35 \mathrm{~g} / \mathrm{l})$ & 1.159 & $0.350-3.840$ & 0.809 \\
\hline $\operatorname{ALT}(>60 \mathrm{U} / 1 \mathrm{vs} . \leq 60 \mathrm{U} / \mathrm{l})$ & 1.352 & $0.547-3.340$ & 0.513 \\
\hline $\operatorname{AST}(>42 \mathrm{U} / 1 \mathrm{vs} . \leq 42 \mathrm{U} / \mathrm{l})$ & 1.859 & $0.856-4.035$ & 0.117 \\
\hline PLT $\left(<100 \times 10^{9} / 1\right.$ vs. $\left.\geq 100 \times 10^{9} / 1\right)$ & 1.992 & $0.918-4.323$ & 0.081 \\
\hline Bilirubin $(>20 \mu \mathrm{mol} / 1 \mathrm{vs} . \leq 20 \mu \mathrm{mol} / \mathrm{l})$ & 1.740 & $0.704-4.302$ & 0.231 \\
\hline Tumor number (multiple vs. single) & 1.799 & $0.683-4.740$ & 0.235 \\
\hline Tumor size $(>5 \mathrm{~cm}$ vs. $\leq 5 \mathrm{~cm})$ & 2.580 & $1.187-5.606$ & $0.017^{\mathrm{a}}$ \\
\hline Tumor margin $(\leq 2 \mathrm{~cm}$ vs. $>2 \mathrm{~cm})$ & 1.052 & $0.476-2.326$ & 0.900 \\
\hline Pathological differentiation (middle and low vs. high) & 1.800 & $0.813-3.987$ & 0.147 \\
\hline Microvascular tumor thrombus (yes vs. no) & 1.347 & $0.609-2.979$ & 0.462 \\
\hline Capsule invasion (yes vs. no) & 1.063 & $0.431-2.623$ & 0.894 \\
\hline TNM stage (III vs. I+II) & 5.364 & $2.123-13.554$ & $<0.001^{\mathrm{a}}$ \\
\hline IL-8 (positive vs. negative) & 11.618 & $4.373-30.863$ & $<0.001^{\mathrm{a}}$ \\
\hline ERK2 (positive vs. negative) & 10.090 & $4.366-23.317$ & $<0.001^{\mathrm{a}}$ \\
\hline IL-8 and ERK2 (both vs. others) & 3.938 & 2.424-6.397 & $<0.001^{\mathrm{a}}$ \\
\hline
\end{tabular}

CI, confidence interval; HBV, hepatitis B virus; AFP, alpha-fetoprotein; ALT, alanine aminotransferase; AST, alanine aminotransferase; PLT, platelet; TNM, tumor, lymph node, metastasis; IL-8, interleukin-8; ERK2, extracellular signal-regulated kinase 2. ${ }^{\mathrm{a}} \mathrm{P}<0.05$.

Relationship between IL-8 and/or ERK2 expression and postoperative prognosis. In the data from the $67 \mathrm{HCC}$ samples, the median follow-up time was $49.56 \pm 25.79$ months (range, 1.8-78.1 months). The OS rates of patients at 1, 3 and 5 years were $85.08,65.67$ and $58.21 \%$, respectively, while DFS rates were 67.16, 49.25 and $40.30 \%$, respectively (Data not shown). Kaplan-Meier survival analysis of 67 patients with HCC exhibited significantly shorter OS $(\mathrm{P}<0.0001$; Fig. 2A) and DFS ( $\mathrm{P}<0.0001$; Fig. 2B) times in the IL- 8 high-expression group compared with the low-expression group. Furthermore, the ERK2 high-expression group had shorter OS ( $\mathrm{P}<0.0001$; Fig. 2C) and DFS ( $\mathrm{P}<0.0001$; Fig. 2D) times than the corresponding low-expression group. Based on these results, the 67 patients with HCC were divided into three groups for further Kaplan-Meier analysis (low IL-8 and low ERK2 group, high IL-8 or high ERK2 group, and high IL-8 and high ERK2 group). OS ( $\mathrm{P}<0.001$; Fig. 3A, C and E) and DFS $(\mathrm{P}<0.0001$; Fig. 3B, D and F) were significantly shorter in the IL- 8 and/or ERK2 high-expression group than in the low-expression group.

Given the significant correlation between IL-8 and ERK2 expression, univariate and multivariate Cox proportional hazard analyses were performed on the IL- 8 expression group, the ERK2 expression group and the IL-8 and ERK2 expression group. Further multivariate Cox regression analysis was then performed on the significant factors identified from univariate analysis (Tables III and IV). The multivariate Cox regression analysis of the IL-8 expression group showed that TNM stage III [hazard ratio $(\mathrm{HR})=6.246 ; 95 \%$ confidence interval (CI), 2.233-17.471; $\mathrm{P}<0.001]$ and high IL-8 expression $(\mathrm{HR}=12.369 ; 95 \% \mathrm{CI}, 4.589-33.341 ; \mathrm{P}<0.001)$ were independent risk factors for OS (Table V), while a platelet count $(\mathrm{PLT})<100 \times 10 \% / 1(\mathrm{HR}=2.106 ; 95 \% \mathrm{CI}, 1.071-4.141$; $\mathrm{P}=0.031)$, TNM stage III $(\mathrm{HR}=3.477$; 95\% CI, 1.367-8.847; $\mathrm{P}=0.009)$ and high-IL- 8 expression $(\mathrm{HR}=6.620 ; 95 \% \mathrm{CI}$, 3.350-13.084; $\mathrm{P}<0.001)$ were all independent risk factors for DFS (Table VI). Multivariate Cox regression analysis of the ERK2 expression group showed that TNM stage III $(\mathrm{HR}=4.832 ; 95 \% \mathrm{CI}, 1.760-13.269 ; \mathrm{P}=0.002)$ and high ERK2 expression $(\mathrm{HR}=10.011 ; 95 \% \mathrm{CI}, 4.268-23.479$; $\mathrm{P}<0.001)$ were independent risk factors for OS (Table V), while PLT $<10010^{9} / 1$ $(\mathrm{HR}=2.171 ; 95 \% \mathrm{CI}, 1.115-4.226 ; \mathrm{P}=0.023)$, TNM stage III $(\mathrm{HR}=2.873 ; 95 \% \mathrm{CI}, 1.153-7.156 ; \mathrm{P}=0.023)$ and high $\mathrm{ERK} 2$ expression $(\mathrm{HR}=5.263$; 95\% CI, 2.760-10.036; $\mathrm{P}<0.001)$ were independent risk factors for DFS (Table VI). Furthermore, multivariate Cox regression models of the IL-8 and ERK2 expression groups showed that TNM stage III (OS: HR=4.595; 95\% CI, 1.682-12.549; $\mathrm{P}=0.003$. DFS: $\mathrm{HR}=2.821 ; 95 \% \mathrm{CI}$, 1.134-7.017; $\mathrm{P}=0.026$ ) with IL-8 and ERK2 co-expression (OS: $\mathrm{HR}=9.082$; 95\% CI, 3.974-20.757; $\mathrm{P}<0.001$. DFS: $\mathrm{HR}=4.918$; 95\% CI, 2.586-9.355; $\mathrm{P}<0.001)$ was an independent risk factor for both OS and DFS (Tables V and VI). PLT<100 10 $/ 1$ $(\mathrm{HR}=2.101 ; 95 \% \mathrm{CI}, 1.080-4.086 ; \mathrm{P}=0.029)$ was also shown to be an independent risk factor for DFS (Table VI). 
Table IV. Univariate analysis of variables with patient disease-free survival (Cox regression model).

\begin{tabular}{|c|c|c|c|}
\hline \multirow[b]{2}{*}{ Variable } & \multicolumn{3}{|c|}{ Univariate analysis } \\
\hline & Hazard ratio & $95 \% \mathrm{CI}$ & P-value \\
\hline Sex (male vs. female) & 1.567 & $0.660-3.719$ & 0.309 \\
\hline Age ( $\geq 50$ vs. $<50$, years $)$ & 1.631 & $0.867-3.069$ & 0.129 \\
\hline HBV (positive vs. negative) & 1.500 & $0.693-3.244$ & 0.304 \\
\hline Cirrhosis (present vs. absent) & 1.380 & $0.190-10.042$ & 0.750 \\
\hline $\operatorname{AFP}(>400 \mu \mathrm{g} / 1 \mathrm{vs} . \leq 400 \mu \mathrm{g} / \mathrm{l})$ & 1.537 & $0.798-2.960$ & 0.198 \\
\hline Albumin $(<35 \mathrm{~g} / \mathrm{l} \mathrm{vs} . \geq 35 \mathrm{~g} / \mathrm{l})$ & 1.008 & $0.360-2.826$ & 0.988 \\
\hline $\operatorname{ALT}(>60 \mathrm{U} / 1 \mathrm{vs} . \leq 60 \mathrm{U} / \mathrm{l})$ & 1.064 & $0.472-2.398$ & 0.882 \\
\hline $\operatorname{AST}(>42 \mathrm{U} / 1 \mathrm{vs} . \leq 42 \mathrm{U} / \mathrm{l})$ & 1.209 & $0.607-2.411$ & 0.589 \\
\hline PLT $\left(<100 \times 10^{9} / 1\right.$ vs. $\left.\geq 100 \times 10^{9} / 1\right)$ & 1.940 & $1.015-3.708$ & $0.045^{\mathrm{a}}$ \\
\hline Bilirubin $(>20 \mu \mathrm{mol} / 1 \mathrm{vs} . \leq 20 \mu \mathrm{mol} / \mathrm{l})$ & 1.400 & $0.648-3.028$ & 0.392 \\
\hline Tumor number (multiple vs. single) & 1.766 & $0.783-3.983$ & 0.171 \\
\hline Tumor size $(>5 \mathrm{~cm}$ vs. $\leq 5 \mathrm{~cm})$ & 2.070 & $1.075-3.987$ & $0.030^{\mathrm{a}}$ \\
\hline Tumor margin $(\leq 2 \mathrm{~cm}$ vs. $>2 \mathrm{~cm})$ & 1.157 & $0.601-2.226$ & 0.663 \\
\hline Pathological differentiation (middle and low vs. high) & 1.085 & $0.532-2.212$ & 0.822 \\
\hline Microvascular tumor thrombus (yes vs. no) & 1.064 & $0.544-2.079$ & 0.856 \\
\hline Capsule invasion (yes vs. no) & 1.088 & $0.520-2.276$ & 0.822 \\
\hline TNM stage (III vs. I+II) & 3.071 & $1.284-7.342$ & $0.012^{\mathrm{a}}$ \\
\hline IL-8 (positive vs. negative) & 6.098 & $3.172-11.720$ & $<0.001^{\mathrm{a}}$ \\
\hline ERK2 (positive vs. negative) & 5.048 & $2.701-9.433$ & $<0.001^{\mathrm{a}}$ \\
\hline IL-8 and ERK2 (both vs. others) & 2.607 & $1.868-3.639$ & $<0.001^{\mathrm{a}}$ \\
\hline
\end{tabular}

CI, confidence interval; HBV, hepatitis B virus; AFP, alpha-fetoprotein; ALT, alanine aminotransferase; AST, alanine aminotransferase; PLT, platelet; TNM, tumor, lymph node, metastasis; IL-8, interleukin-8; ERK2, extracellular signal-regulated kinase 2. ${ }^{\mathrm{a}} \mathrm{P}<0.05$.

\section{Discussion}

The present study demonstrated that HCC patients with high IL-8 and/or ERK2 expression had significantly higher risks of recurrence and death than those with low expression. The Oncomine ${ }^{\mathrm{TM}}$ database and IHC were used to show that IL-8 and ERK2 expression were significantly higher in HCC tissues compared with non-tumor liver tissues. A positive correlation was also found between IL-8 and ERK2 expression in tissues from HCC patients using RT-qPCR. Moreover, IL-8 and/or ERK2 expression was significantly associated with tumor size and differentiation, and patients with high IL-8 and/or ERK2 expression had a poorer prognosis than the low expression group. Multivariate survival analysis further supported the high expression of IL-8 and/or ERK2 in HCC as an independent risk factor for OS and DFS.

The important role played by inflammation and the immune response in the occurrence and development of tumors has gradually become more recognized. A previous study reported that IL-8 is closely associated with the occurrence and development of HCC (25). In the present study, it was observed that IL-8 was expressed to a significantly higher level in HCC tissues, compared with non-tumor liver tissues, consistent with the results of a previous study (14). Schmitz et al (19) detected high expression levels of ERK1/2 in patient HCC tissues, and this was also detected in the HCC tissues examined in the present study. Waugh and Wilson (16) reported that IL-8 in tumor cells was able to promote ERK1/2 phosphorylation,
Table V. Multivariate analysis of variables with patient overall survival (Cox regression model).

\begin{tabular}{lrrr}
\hline & $\begin{array}{c}\text { Hazard } \\
\text { Variable }\end{array}$ & & \\
\hline IL-8 & & & \\
$\quad$ TNM stage (III vs. I+II) & 6.246 & $2.233-17.471$ & $<0.001^{\mathrm{a}}$ \\
IL-8 (high vs. low) & 12.369 & $4.589-33.341$ & $<0.001^{\mathrm{a}}$ \\
ERK2 & & & \\
TNM stage (III vs. I+II) & 4.832 & $1.760-13.269$ & $0.002^{\mathrm{a}}$ \\
$\begin{array}{l}\text { ERK2 (high vs. low) } \\
\text { IL-8 and ERK2 }\end{array}$ & 10.011 & $4.268-23.479$ & $<0.001^{\mathrm{a}}$ \\
TNM stage (III vs. I+II) & 4.595 & $1.682-12.549$ & $0.003^{\mathrm{a}}$ \\
$\begin{array}{l}\text { IL-8 and ERK2 } \\
\text { (both vs. others) }\end{array}$ & 9.082 & $3.974-20.757$ & $<0.001^{\mathrm{a}}$ \\
\hline
\end{tabular}

CI, confidence interval; TNM, tumor, lymph node, metastasis; IL-8, interleukin-8; ERK2, extracellular signal-regulated kinase 2. ${ }^{\mathrm{a}} \mathrm{P}<0.05$.

thereby promoting tumor growth and metastasis. A significant positive correlation between IL- 8 and ERK2 in HCC tissues was also detected, indicating that high expression of IL-8 influences disease progression by activating ERK2. 
Table VI. Multivariate analysis of variables with patient disease-free survival (Cox regression model).

\begin{tabular}{|c|c|c|c|}
\hline Variable & $\begin{array}{c}\text { Hazard } \\
\text { ratio }\end{array}$ & $95 \% \mathrm{CI}$ & P-value \\
\hline \multicolumn{4}{|l|}{ IL-8 } \\
\hline $\begin{array}{l}\text { PLT }\left(<100 \times 10^{9} / 1 \text { vs. }\right. \\
\left.\geq 100 \times 10^{9} / 1\right)\end{array}$ & 2.106 & $1.071-4.141$ & $0.031^{\mathrm{a}}$ \\
\hline TNM stage (III vs. I+II) & 3.477 & $1.367-8.847$ & $0.009^{\mathrm{a}}$ \\
\hline IL-8 (high vs. low) & 6.620 & $3.350-13.084$ & $<0.001^{\mathrm{a}}$ \\
\hline \multicolumn{4}{|l|}{ ERK2 } \\
\hline $\begin{array}{l}\operatorname{PLT}\left(<100 \times 10^{9} / 1 \text { vs. }\right. \\
\left.\geq 100 \times 10^{9} / 1\right)\end{array}$ & 2.171 & $1.115-4.226$ & $0.023^{\mathrm{a}}$ \\
\hline TNM stage (III vs. I+II) & 2.873 & $1.153-7.156$ & $0.023^{\mathrm{a}}$ \\
\hline ERK2 (high vs. low) & 5.263 & $2.760-10.036$ & $<0.001^{\mathrm{a}}$ \\
\hline \multicolumn{4}{|l|}{ IL-8 and ERK2 } \\
\hline $\begin{array}{l}\text { PLT }\left(<100 \times 10^{9} / 1 \mathrm{vs}\right. \\
\left.\geq 100 \times 10^{9} / 1\right)\end{array}$ & 2.101 & $1.080-4.086$ & $0.029^{\mathrm{a}}$ \\
\hline TNM stage (III vs. I+II) & 2.821 & $1.134-7.017$ & $0.026^{\mathrm{a}}$ \\
\hline $\begin{array}{l}\text { IL-8 and ERK2 } \\
\text { (both vs. others) }\end{array}$ & 4.918 & $2.586-9.355$ & $<0.001^{\mathrm{a}}$ \\
\hline
\end{tabular}

CI, confidence interval; PLT, platelet; TNM, tumor, lymph node, metastasis; IL-8, interleukin-8; ERK2, extracellular signal-regulated kinase $2 .{ }^{\mathrm{a}} \mathrm{P}<0.05$.

There is increasing evidence that serum IL- 8 levels are an effective predictor of prognosis in patients with HCC (26), pancreatic cancer (27) and lymphoma (28), and that high nuclear ERK2 expression is an indicator of poor prognosis in patients with invasive breast cancer (29). However, these studies have been limited to the use of serum IL-8, and the relationship between IL-8 and/or ERK2 expression in HCC tissues and patient prognosis is poorly understood. The present study found that patients with high expression levels of IL-8 and/or ERK2 in HCC tissues had a worse prognosis than those in the low expression group. In addition, high expression levels of IL-8 and/or ERK2 in HCC tissues were shown to be an independent risk factor for OS and DFS in HCC. These data provide new insights for research into HCC progression, which may offer novel targets for the development of anti-tumor drugs.

The cytokine network is a complex system, with cytokine gene expression affected by other cytokines and the existence of synergistic restrictions and inhibition $(30,31)$. As well as elevated IL-8 levels, patients with HCC also exhibit characteristic changes in expression of various cytokines, such as decreased IL-2 or elevated IL- 6 and tumor necrosis factor- $\alpha$ (32-34). Therefore, in addition to the application of IL-8 and ERK2 as early indicator molecules, the deregulation of other cytokines in HCC should be examined to advance disease understanding, diagnosis and treatment. Moreover, because the present study is a single-center study of a small sample size, future studies of larger sample populations should be investigated to confirm these findings.

In summary, a significant positive correlation between the expression of IL-8 and ERK2 in HCC tissues was reported.
It was also found that high expression levels of IL-8 and/or ERK2 in HCC tissues were an independent risk factor for OS and DFS, and that HCC patients with high IL-8 and/or ERK2 expression had worse prognoses than those with low expression. The results of the present study support the hypothesis that patients with high expression of IL-8 and/or ERK2 should undergo more frequent follow-ups. Additionally, IL-8 and ERK2 are potential predictors of postoperative prognosis in patients with HCC, and therefore, may be used as therapeutic targets for the development of drugs that prevent HCC recurrence, thereby improving the long-term survival of patients with HCC.

\section{Acknowledgements}

The authors would like to thank Dr Sarah Williams (University of Oxford, Oxford, UK) for her assistance in revising this manuscript.

\section{Funding}

The present study was supported by the Hunan Provincial Natural \& Science Foundation (grant no. 2018JJ6126).

\section{Availability of data and materials}

The datasets used and/or analyzed during the present study are available from the corresponding author upon reasonable request.

\section{Authors' contributions}

Study conception and design were conducted by GH, who also provided administrative support. YD, QY and $\mathrm{BH}$ provided the study materials. YD, QY, BH and ZH collected and assembled the data, and data analysis and interpretation were undertaken by YD and ZN. The manuscript was written and approved by all of the authors.

\section{Ethics approval and consent to participate}

The use of materials in the present study was approved by the Hunan Normal University Medical Ethics Committee, and all patients provided written informed consent.

\section{Patient consent for publication}

Not applicable.

\section{Competing interests}

The authors declare that they have no competing interests.

\section{References}

1. Gao J, Xie L, Yang WS, Zhang W, Gao S, Wang J and Xiang YB: Risk factors of hepatocellular carcinoma-Current status and perspectives. Asian Pac J Cancer Prev 13: 743-752, 2012.

2. Bray F, Ferlay J, Soerjomataram I, Siegel RL, Torre LA and Jemal A: Global cancer statistics 2018: GLOBOCAN estimates of incidence and mortality worldwide for 36 cancers in 185 countries. CA Cancer J Clin 68: 394-424, 2018. 
3. Chen W, Zheng R, Baade PD, Zhang S, Zeng H, Bray F, Jemal A, Yu XQ and He J: Cancer statistics in China, 2015. CA Cancer J Clin 66: 115-132, 2016

4. Hong J, Hu K, Yuan Y, Sang Y, Bu Q, Chen G, Yang L, Li B, Huang $\mathrm{P}$, Chen $\mathrm{D}$, et al: CHK1 targets spleen tyrosine kinase (L) for proteolysis in hepatocellular carcinoma. J Clin Inves 122: 2165-2175, 2012.

5. Cha C, Fong Y, Jarnagin WR, Blumgart LH and DeMatteo RP: Predictors and patterns of recurrence after resection of hepatocellular carcinoma. J Am Coll Surg 197: 753-758, 2003.

6. Bosch FX, Ribes J, Diaz M and Cleries R: Primary liver cancer: Worldwide incidence and trends. Gastroenterology 127: S5-S16, 2004.

7. Lin WW and Karin M: A cytokine-mediated link between innate immunity, inflammation, and cancer. J Clin Invest 117: $1175-1183,2007$.

8. Zlotnik A and Yoshie O: Chemokines: A new classification system and their role in immunity. Immunity 12: 121-127, 2000.

9. Yuan A, Chen JJ, Yao PL and Yang PC: The role of interleukin-8 in cancer cells and microenvironment interaction. Front Biosci 10: 853-865, 2005 .

10. Lazennec G and Richmond A: Chemokines and chemokine receptors: New insights into cancer-related inflammation. Trends Mol Med 16: 133-144, 2010.

11. Zhang Y, Wang L, Zhang M, Jin M, Bai C and Wang X: Potential mechanism of interleukin-8 production from lung cancer cells: An involvement of EGF-EGFR-PI3K-Akt-Erk pathway. J Cell Physiol 227: 35-43, 2012.

12. Mohamed MM: Monocytes conditioned media stimulate fibronectin expression and spreading of inflammatory breast cancer cells in three-dimensional culture: A mechanism mediated by IL-8 signaling pathway. Cell Commun Signal 10: 3, 2012.

13. Ning Y, Labonte MJ, Zhang W, Bohanes PO, Gerger A, Yang D, Benhaim L, Paez D, Rosenberg DO, Nagulapalli Venkata KC, et al: The CXCR 2 antagonist, SCH-527123, shows antitumor activity and sensitizes cells to oxaliplatin in preclinical colon cancer models. Mol Cancer Ther 11: 1353-1364, 2012.

14. Wang Y, Wang W, Wang L, Wang X and Xia J: Regulatory mechanisms of interleukin- 8 production induced by tumour necrosis factor- $\alpha$ in human hepatocellular carcinoma cells. J Cell Mol Med 16: 496-506, 2012.

15. Harimoto N, Shirabe K, Abe T, Kajiyama K, Nagaie T, Gion T, Kuroda Y and Maehara Y: Interleukin-8 producing hepatocellular carcinoma with pyrexia. HPB Surg 2009: 461492, 2009.

16. Waugh DJ and Wilson C: The interleukin-8 pathway in cancer. Clin Cancer Res 14: 6735-6741, 2008.

17. Cowley S, Paterson H, Kemp P and Marshall CJ: Activation of MAP kinase kinase is necessary and sufficient for PC12 differentiation and for transformation of NIH 3T3 cells. Cell 77: 841-852, 1994.

18. Mansour SJ, Matten WT, Hermann AS, Candia JM, Rong S, Fukasawa K, Vande Woude GF and Ahn NG: Transformation of mammalian cells by constitutively active MAP kinase kinase. Science 265: 966-970, 1994.

19. Schmitz KJ, Wohlschlaeger J, Lang H, Sotiropoulos GC, Malago M, Steveling K, Reis H, Cicinnati VR, Schmid KW and Baba HA: Activation of the ERK and AKT signalling pathway predicts poor prognosis in hepatocellular carcinoma and ERK activation in cancer tissue is associated with hepatitis $\mathrm{C}$ virus infection. J Hepatol 48: 83-90, 2008.

20. Mas VR, Maluf DG, Archer KJ, Yanek K, Kong X, Kulik L, Freise CE, Olthoff KM, Ghobrial RM, McIver P and Fisher R: Genes involved in viral carcinogenesis and tumor initiation in hepatitis $C$ virus-induced hepatocellular carcinoma. Mol Med 15: 85-94, 2009.
21. Roessler S, Jia HL, Budhu A, Forgues M, Ye QH, Lee JS, Thorgeirsson SS, Sun Z, Tang ZY, Qin LX and Wang XW: A unique metastasis gene signature enables prediction of tumor relapse in early-stage hepatocellular carcinoma patients. Cancer Res 70: 10202-10212, 2010

22. Kamarajah SK, Frankel TL, Sonnenday C, Cho CS and Nathan H: Critical evaluation of the American Joint Commission on Cancer (AJCC) 8th edition staging system for patients with Hepatocellular Carcinoma (HCC): A surveillance, epidemiology, end results (SEER) analysis. J Surg Onco 117: 644-650, 2018.

23. He L, Fan X, Li Y, Chen M, Cui B, Chen G, Dai Y, Zhou D, Hu X and Lin H: Overexpression of zinc finger protein 384 (ZNF 384), a poor prognostic predictor, promotes cell growth by upregulating the expression of Cyclin D1 in Hepatocellular carcinoma. Cell Death Dis 10: 444, 2019.

24. Livak KJ and Schmittgen TD: Analysis of relative gene expression data using real-time quantitative PCR and the 2(-Delta Delta C(T)) method. Methods 25: 402-408, 2001.

25. Ren Y, Poon RT, Tsui HT, Chen WH, Li Z, Lau C, Yu WC and Fan ST: Interleukin-8 serum levels in patients with hepatocellular carcinoma: Correlations with clinicopathological features and prognosis. Clin Cancer Res 9: 5996-6001, 2003.

26. Loosen SH, Schulze-Hagen M, Leyh C, Benz F, Vucur M, Kuhl C, Trautwein C, Tacke F, Bruners P, Roderburg C and Luedde T: IL-6 and IL-8 serum levels predict tumor response and overall survival after TACE for primary and secondary hepatic malignancies. Int J Mol Sci 19: pii: E1766, 2018.

27. Feng L, Qi Q, Wang P, Chen H, Chen Z, Meng Z and Liu L: Serum levels of IL-6, IL-8, and IL-10 are indicators of prognosis in pancreatic cancer. J Int Med Res 46: 5228-5236, 2018.

28. Nacinovic-Duletic A, Stifter S, Dvornik S, Skunca Z and Jonjic N: Correlation of serum IL-6, IL-8 and IL-10 levels with clinicopathological features and prognosis in patients with diffuse large B-cell lymphoma. Int J Lab Hematol 30: 230-239, 2008.

29. Nakopoulou L, Mylona E, Rafailidis P, Alexandrou $P$, Giannopoulou I and Keramopoulos A: Effect of different ERK2 protein localizations on prognosis of patients with invasive breast carcinoma. APMIS 113: 693-701, 2005.

30. Becher B, Spath S and Goverman J: Cytokine networks in neuroinflammation. Nature reviews. Immunology 17: 49-59, 2017.

31. Morel PA, Lee REC and Faeder JR: Demystifying the cytokine network: Mathematical models point the way. Cytokine 98: 115-123, 2017.

32. Liu XL, Li FQ, Liu LX, Li B and Zhou ZP: TNF- $\alpha$, HGF and macrophage in peritumoural liver tissue relate to major risk factors of HCC Recurrence. Hepatogastroenterology 60: 1121-1126, 2013.

33. Peng Q, Li H, Lao X, Deng Y, Chen Z, Qin X and Li S: Association of IL-2 polymorphisms and IL-2 serum levels with susceptibility to HBV-related hepatocellular carcinoma in a Chinese Zhuang population. Infect Genet Evol 27: 375-381, 2014.

34. Pine SR, Mechanic LE, Enewold L, Chaturvedi AK, Katki HA, Zheng YL, Bowman ED, Engels EA, Caporaso NE and Harris CC: Increased levels of circulating interleukin 6, interleukin 8, C-reactive protein, and risk of lung cancer. J Natl Cancer Inst 103: 1112-1122, 2011.

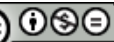

This work is licensed under a Creative Commons Attribution-NonCommercial-NoDerivatives 4.0 International (CC BY-NC-ND 4.0) License. 